\title{
Dimension of spatially embedded networks
}

\author{
Li Daqing ${ }^{1 \star}$, Kosmas Kosmidis ${ }^{2,3}$, Armin Bunde ${ }^{2}$ and Shlomo Havlin ${ }^{1}$
}

The dimension of a system is one of the most fundamental quantities to characterize its structure and basic physical properties. Diffusion' and vibrational excitations ${ }^{2}$, for example, as well as the universal features of a system near a critical point depend crucially on its dimension ${ }^{3,4}$. However, in the theory of complex networks the concept of dimension has been rarely discussed. Here we study models for spatially embedded networks and show how their dimension can be determined. Our results indicate that networks characterized by a broad distribution of link lengths have a dimension higher than that of the embedding space. We illustrate our findings using the global airline network and the Internet and argue that although these networks are embedded in two-dimensional space they should be regarded as systems with dimension close to 3 and 4.5, respectively. We show that the network dimension is a key concept to understand not only network topology, but also dynamical processes on networks, such as diffusion and critical phenomena including percolation.

Networks consist of entities (nodes) and their connections (links) ${ }^{5}$. Usually, networks are embedded either in two- or in threedimensional space. For example, airline and Internet networks, as well as friendship networks where the nodes are the residences of friends, are embedded in the two-dimensional surface of the earth, whereas the neuronal network in the brain is embedded in a complex three-dimensional structure. If in a $d$-dimensional lattice, the links connect only neighbouring nodes (in space), then the dimension of the network is trivially identical to the dimension of the embedding space. In most cases, however, links are not short ranged, their length distribution is broad, connecting also distant nodes. The question we pose here is: Is there a finite dimension that characterizes such a spatially embedded network and how can we determine it? The knowledge of the dimension ${ }^{6,7}$ is not only important for a structural characterization of the network, but is also crucial for understanding the function of the network, as the dimension governs the dynamical processes in the network.

In network theory, the dimension of a network has been rarely considered. Research ${ }^{8-13}$ has focused on two types of networks where the links between the nodes are either short range, connecting only nearby nodes (like a lattice), or long range, connecting any two nodes with the same probability. In the short range case, the dimension $d$ of the network is identical to the dimension of the embedding space, whereas in the long range case the embedding space is irrelevant and the network can be regarded as having an infinite dimension. As a consequence, the mean distance between two nodes scales with the number of nodes $N$ as $N^{1 / d}$ in the short range case, and as $\log N$ (ref. 14) or $\log \log N$ (ref. 15) in the long range case, reflecting its 'small world' or 'ultra small world' character, respectively. Examples of long range networks include the classical Bethe and Erdös-Rényi ${ }^{16,17}$ networks and the more recent Watts-Strogatz ${ }^{18}$ and Barabási-Albert models ${ }^{19}$.

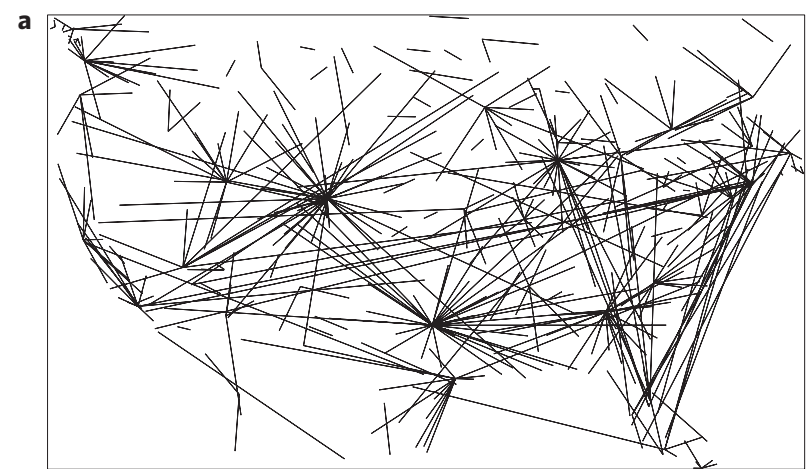

b

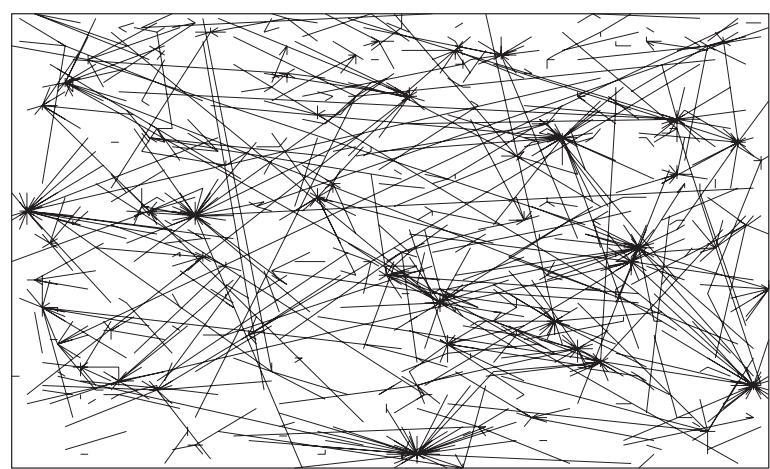

Figure 1 | Spatially embedded real and model networks. a, The airline network in the USA region. The airports are linked together by direct flights. For clarity, only flights with more than 200,000 seats for the summer of 2008 are shown. b. Model network with the same parameters as the airline network ( $\delta=3$ and $\alpha=1.8$ ). Note the similarity between model and real data.

Many real networks, however, do not fall into these categories, and the lengths of their links are characterized by a broad power law distribution. For example, in a mobile phone communication network, the probability $P(r)$ to have a friend at distance $r$ decays as $P(r) \sim r^{-2}$ (ref. 20), and in the global airline network, the probability that an airport has a link (direct flight) to an airport at distance $r$, decays as $P(r) \sim r^{-3}$ (ref. 21) (see Fig. 1a). By definition, short range and long range networks correspond to $P(r) \sim r^{-\delta}$, with $\delta=\infty$ and $\delta=0$, respectively.

A fundamental open question is whether also spatially embedded networks with $0<\delta<\infty$ can be characterized by a dimension. Here we show how the dimension of those networks can be determined and that it plays a basic role, not only in characterizing the structure, but also in determining the dynamical properties on the network and its behaviour near a critical point.

\footnotetext{
${ }^{1}$ Minerva Center and Department of Physics, Bar Ilan University, Ramat Gan 52900, Israel, ${ }^{2}$ Institut für Theoretische Physik III, Justus Liebig Universität Giessen, Giessen, 35392, Germany, ${ }^{3}$ Physics Department and Network Operations Center, Aristotle University of Thessaloniki, Thessaloniki, 54124, Greece. *e-mail: li.daqing.biu@gmail.com.
} 

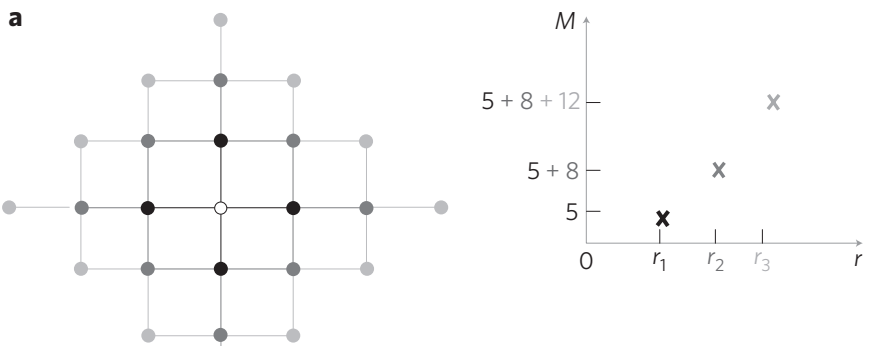

b
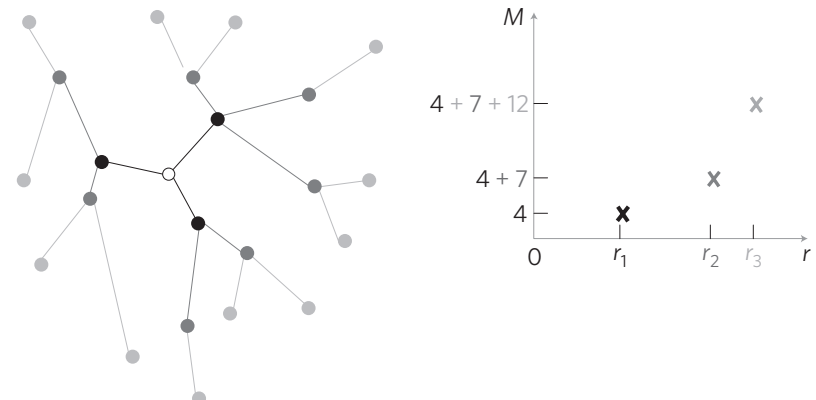

Figure 2 | Determination of the network dimension. a for a regular lattice and $\mathbf{b}$ for a complex network. The figures show, around a randomly chosen node (open circle), shell 1 (black), shell 2 (dark grey) and shell 3 (light grey). On the right-hand side, the number of nodes $M$ is plotted versus the mean spatial distance $r$ of each shell from the origin site. From the scaling relation $M \sim r^{d}$ (equation (1)), we obtain the network dimension $d$.

To construct a model of spatially embedded networks, we start with a network whose nodes are the sites of a $d$-dimensional regular lattice. To generate the links between them, we choose a node $i$ and determine its number of links (degree) $k_{i}$ from a given degree distribution $P(k)$. Then we select, for each of these links, a distance $r$ from a given probability distribution $P(r)$, pick randomly one of the available nodes at Euclidean distance $r$ from node $i$ and connect both. We repeat this process for all nodes and available links in the network and remove multiple connections ${ }^{22}$. A typical realization of such a network, with the same parameters as for the airline network in Fig. 1a, is shown in Fig. 1b. In contrast to lattices, all length scales of links occur.

Next we show how the dimension $d$ of the network can be obtained in the general case. We use the fact that the mass $M$ (here: number of nodes) of an object within an hypersphere of radius $r$ scales with $r$ as

$$
M \sim r^{d}
$$

where the exponent $d$ represents the dimension of the network. If we use this relation without taking into account the way the nodes are linked, we trivially find the incorrect result that the dimension of the network is identical to the dimension of the embedding space.

To properly take into account the connectivity of the network, we suggest the following procedure: we consider, for a node chosen as origin (open symbols in Fig. 2a,b), its nearest neighbours (referred to shell $l=1)$, its next nearest neighbours $(l=2)$, and so on. Next we measure (1) the mean Euclidean distance $r(l)$ of the nodes in shell $l$ from the origin and (2) the number of nodes $M(l)$ within shell $l$. By definition, $l$ is the length of the shortest path between the node in the origin and any node in shell $l$. This procedure is illustrated in Fig. 2, for both a square lattice and a general embedded network. To improve the statistics, we repeat the calculations for many origin nodes, and then average $r(l)$ and $M(l)$. From the scaling relation between the average $M$ and the average $r$, equation (1), we determine the dimension of the spatially embedded network. One can easily verify that, for regular lattices, this procedure gives the expected value of the lattice dimension $d$. It can be also used to measure fractal dimensions ${ }^{7,23}$.

In the following, we apply equation (1) to determine the dimension of spatially embedded networks. Here we analyse two types of spatially embedded networks. Erdös-Rényi networks with Poissonian degree distribution $\left(P(k) \sim \lambda^{k} / k !\right)$ and scale-free networks with power law degree distribution $\left(P(k) \sim k^{-\alpha}\right)$. Representative results of $M(r)$ for spatially embedded Erdös-Rényi and scale-free networks are shown in Fig. 3a,b. The straight lines in the double logarithmic presentation support equation (1), and the slopes of the lines yield the dimensions of the networks (see Table 1). It is interesting to note that the dimension seems to be controlled mainly by $\delta$ and does not seem to be influenced by the degree distribution, indicating a universal feature of the dimension. This feature is further illustrated in the Supplementary Information, where we give further evidence that the dimension does not depend on the size of the network and is independent of the average degree $\langle k\rangle$.

Figure $3 c, d$ shows that the network dimensions obtained in Fig. 3a,b play a fundamental role in physical processes such as diffusion ${ }^{2,24,25}$. The graphs show, for the same networks as in Fig. 3a,b, the probability $P_{0}$ that a diffusing particle, after having traveled $t$ steps, has returned to the origin. As we show below using general arguments, $P_{0}$ scales as

$$
P_{0} \sim r^{-d}
$$

where $r=r(t)$ in this case is the root mean square (r.m.s) displacement of the particle at time $t$. The r.m.s. displacement of a diffusing particle at time $t, r(t)$, characterizes the distance from its position at $t=0$. To derive equation (2), it is assumed that the probability of the particle to be in any site in the volume $V=r(t)^{d}$ visited by the particle is the same. From this it follows that the probability of being at the origin is proportional to $1 / V$, which leads to the scaling relation, equation (2). Figure $3 c, d$ shows that this fundamental relation is indeed valid for both types of spatially embedded networks.

Next we consider how the critical behaviour of the network depends on the dimension. As an example we choose percolation ${ }^{26}$, which is perhaps the most important critical phenomenon, with applications ranging from oil recovery and transport properties of materials to the robustness of networks and the spreading of epidemics. In percolation, a fraction $q$ of nodes is removed from the network. At a critical fraction $q_{\mathrm{c}}$, the network disintegrates and finite clusters of all sizes, $s$, are formed. A general result of percolation theory is that, at criticality, the size distribution, $n(s)$, obeys a power law, $n(s) \sim s^{-\tau}$, where the exponent

$$
\tau=1+\frac{d}{d_{\mathrm{f}}}
$$

is related to the dimension $d$ of the network (obtained from equation (1), see Fig. 3a,b) and the fractal dimension $d_{\mathrm{f}}$ of the network clusters at $q_{\mathrm{c}}$ (ref. 26).

Figure $3 \mathrm{e}$ shows, for two representative networks, the fractal dimensions $d_{\mathrm{f}}$ of these percolation clusters, obtained from the slopes of $M(r)$ in the double logarithmic presentation. Figure $3 \mathrm{f}$ shows, for the same networks, the cluster size distribution, $n(s)$, at criticality, again in a double logarithmic presentation. From the slopes of the straight lines we obtain the values of $\tau$ (see Table 1 ). The results show that the fundamental relation (3) is only valid when, for $d$, the values of the network dimension obtained above are substituted (see Table 1). This reveals the important role of the network dimension in the percolation process.

Finally, we demonstrate that the network dimension plays a major role in real systems such as the global airline network ${ }^{27}$ 

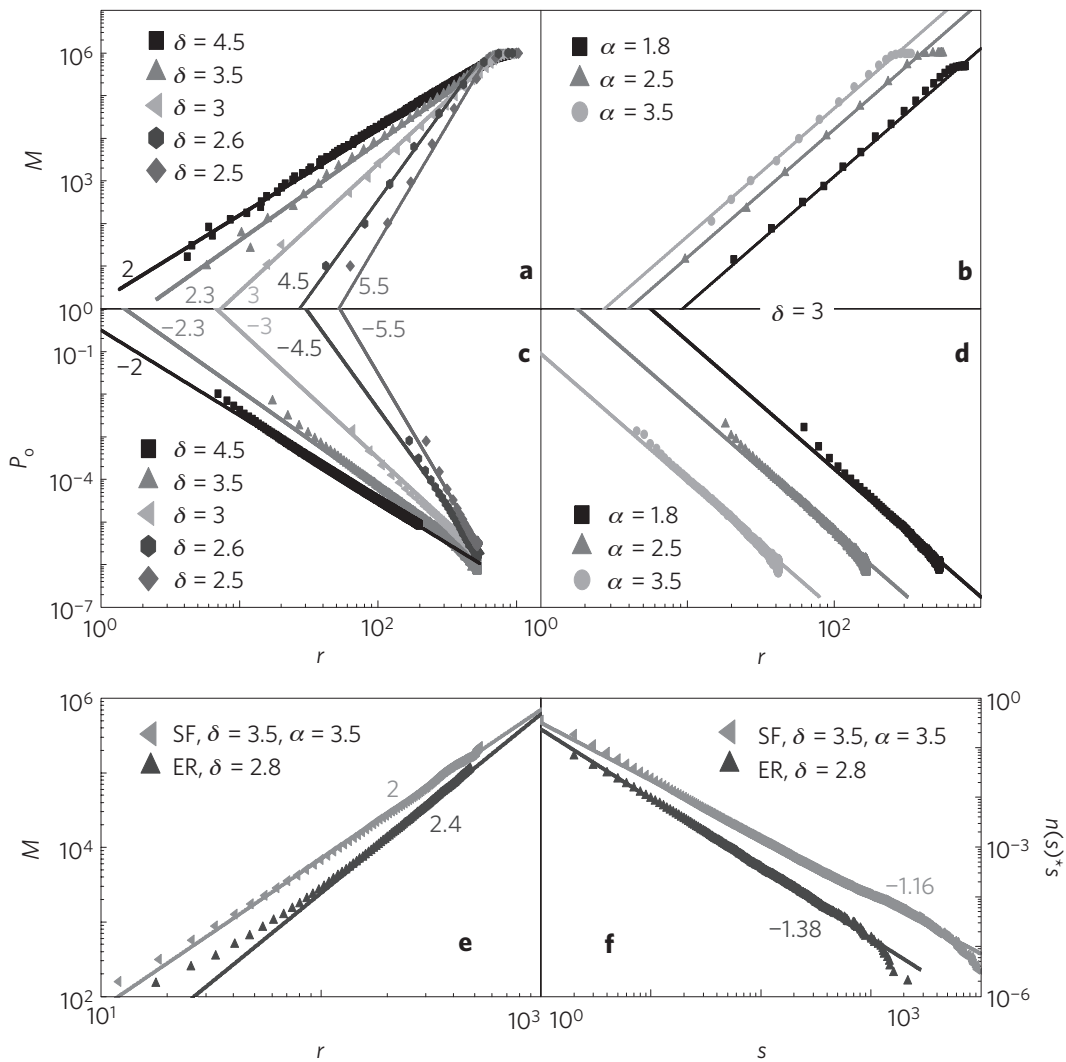

Figure 3 | Dimension and its relation to diffusion and percolation. The scaling relations between the mass $M$ and the metric distance $r$ for $\mathbf{a}$ Erdös-Rényi (ER) networks with a Poissonian degree distribution and $\mathbf{b}$ scale-free (SF) networks with a power law degree distribution $\left(P(k) \sim k^{-\alpha}\right)$. In $\mathbf{a}$, the distance exponent $\delta$ varies between 2.5 and 4.5. $\ln \mathbf{b}, \delta=3$ and the degree exponent $\alpha$ varies between 1.8 and 3.5. The figures show that the network dimension for Erdös-Rényi networks increases monotonically with decreasing $\delta$. For $\delta=3$, the network dimensions in Erdös-Rényi and scale-free networks seem to coincide. $\mathbf{c}, \mathbf{d}$ show for the same networks as in $\mathbf{a}, \mathbf{b}$ respectively, the probability that a diffusing particle is at its starting site, after travelling an average distance $r$. The figure shows that the negative slopes representing the dimension in equation (1) in the double logarithmic plots agree with the network dimension obtained by equation (2). e,f, show, for two representative scale-free and Erdös-Rényi networks at the percolation threshold, the fractal dimension $d_{f}$ (obtained from the slopes in $\mathbf{e}$ ) and the exponent $\tau$ (obtained from the slopes of the cluster size distribution in $\mathbf{f}$ ). The figures show that $d_{f}$ and $\tau$ are related via the network dimension $d: \tau=1+d / d_{f}$.

and the Internet. The Internet we study here has been mapped into a city network where a node represents a city and a link is established when there is a direct router connection between two cities ${ }^{28}$. Both networks are embedded in two-dimensional space. The airline network is characterized by $\delta \simeq 3$ and has a scale-free degree distribution $P(k) \sim k^{-\alpha}$ with $\alpha \simeq 1.8$ (ref. 29), whereas the Internet is characterized by $\delta \simeq 2.6$ and has a power law degree distribution with $\alpha \simeq 2.1$ (see Supplementary Information). Model networks with these features are shown in Fig. 3, predicting a dimension close to 3 for the airline network and close to 4.5 for the Internet. Indeed, when measuring directly the dimension of the airline network and the Internet using equations (1) and (2) we obtain $d \simeq 3$ for the airline network (see Fig. 4a,b) and $d \simeq 4.5$ for the Internet (see Fig. 4c,d).

In summary, our results indicate that spatially embedded networks have an underlying dimension that characterizes relevant physical processes. Throughout the paper we have studied networks that are embedded in two-dimensional space, with a power law distribution of link lengths, $P(r) \sim r^{-\delta}$. Prominent examples are the global airline network and the Internet, which are characterized by $\delta \simeq 3$ and 2.6, respectively. We find that the exponent $\delta$ plays an important role in determining the network dimension. For $\delta>4$, the link lengths are mainly short and the dimension of the network is two, the same as the embedding lattice (see Table 1). Networks with an exponential distribution of link lengths fall into the same universality class; an example is the European

\section{Table 1 | Network dimensions and critical exponents for} Erdös-Rényi networks.

\begin{tabular}{llllll}
$\boldsymbol{\delta}$ & $\boldsymbol{d}$ (equation (1)) & $\boldsymbol{d}$ (equation (2)) & $\boldsymbol{d}$ (equation (3)) & $\boldsymbol{\tau}$ & $\boldsymbol{d}_{\boldsymbol{f}}$ \\
\hline 4.5 & 2.0 & 2.0 & 2.0 & 2.05 & 1.9 \\
3.5 & 2.3 & 2.3 & 2.3 & 2.16 & 2.0 \\
3.3 & 2.5 & 2.5 & 2.5 & 2.21 & 2.1 \\
3.0 & 3.0 & 3.1 & 3.0 & 2.36 & 2.2 \\
2.8 & 3.3 & 3.4 & 3.3 & 2.38 & 2.4 \\
2.5 & 5.5 & 5.7 & 5.6 & 2.48 & 3.8 \\
\hline
\end{tabular}

The values of $d$ in the second column have been obtained by direct measurement of $d$ using equation (1). The values of $d$ in the third column have been obtained from equation (2) by measuring the probability $P_{0}$ of being at the origin after travelling a distance $r$. The values of $d$ in the fourth column have been obtained from equation (3) by measuring $\tau$ and $d_{\mathrm{f}}$ at the percolation threshold. Note that, within the error bars, the three methods yield the same result for dimension $d$. The error bars, which have been analysed by simulating many realizations, are of the order of the last specified digit.

power grid (see Supplementary Information). On the contrary, for $\delta<2$, the network dimension becomes infinite with no constraints of the embedding space. In between, for $\delta$ between two and four, the spatial constraints lead to a dimension of the network that increases monotonically with decreasing $\delta$, from $d=2$ to $\infty$. Our results for the global airline network, the Internet and the European power grid are in agreement with these 
a

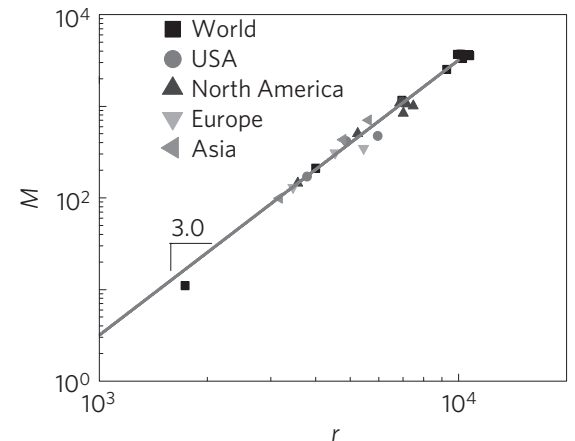

b

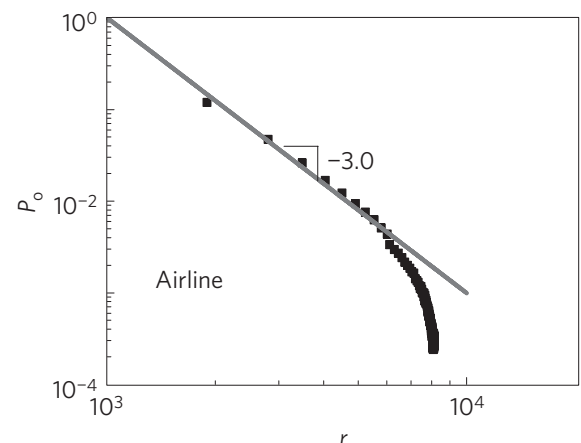

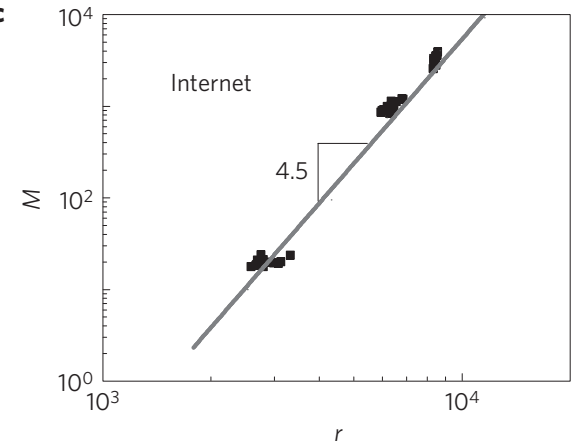

d

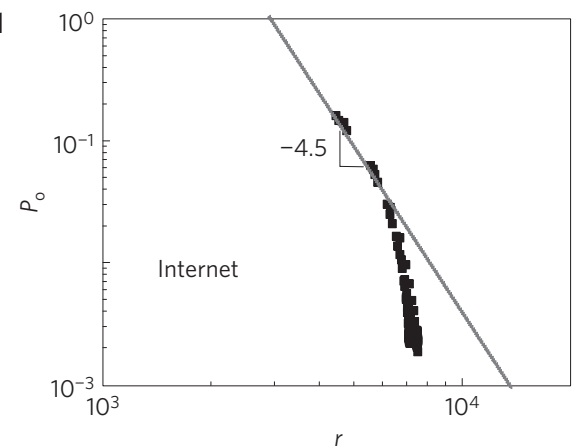

Figure 4 | Dimension and its relation with diffusion in the global airline network and the Internet. a,c, The number of nodes $M$ as a function of the metric distance $r$ in a the global airline network for different regions: worldwide, USA, North America, Europe and Asia and in c the Internet (August 2009 to December 2009). b,d, The diffusion process on the airline network and the Internet, respectively. Measured is the probability of being at the origin $P_{0}$, as a function of the root mean square displacement $r$ of the diffusing particles. The sharp decay for large $r$ is due to the finite size of the considered networks, which is also seen in the model network (see Supplementary Fig. S5).

conclusions. We have focused here on link length distributions $P(r)$ that follow a power law, but other possibilities could also be considered. However, it is clear that, if the second moment of $P(r)$ is finite (as for the exponential distribution of the power grid), the network is well constrained and has the same dimension as its embedding space. In some cases, the assumption of a hyperbolic geometry instead of the Euclidean space may also be applicable ${ }^{30}$.

\section{Received 20 July 2010; accepted 19 January 2011; published online} 27 February 2011

\section{References}

1. Weiss, G. H. Aspects and Applications of the Random Walk (North Holland, 1994).

2. Alexander, S. \& Orbach, R. Density of states on fractals. J. Phys. Lett. 43, 625-631 (1982).

3. Cardy, J. Scaling and Renormalization in Statistical Physics (Cambridge Univ. Press, 1996).

4. Plischke, M. \& Bergersen, B. Equilibrium Statistical Physics (World Scientific, 1994).

5. Barabási, A-L. Linked (Perseus Publishing, 2002).

6. Mandelbrot, B. B. The Fractal Geometry of Nature (Freeman, 1982)

7. Vicsek, T. Fractal Growth Phenomena (World Scientific, 1992).

8. Albert, R. \& Barabási, A-L. Statistical mechanics of complex networks. Rev. Mod. Phys. 74, 47-97 (2002).

9. Newman, M. E. J. The structure and function of complex networks. SIAM Rev. 45, 167-256 (2003).

10. Boccaletti, S., Latora, V., Moreno, Y., Chavez, M. \& Hwang, D. U. Complex networks: Structure and dynamics. Phys. Rep. 424, 175-308 (2006).

11. Dorogovtsev, S. N., Goltsev, A. V. \& Mendes, J. F. F. Critical phenomena in complex networks. Rev. Mod. Phys. 80, 1275-1335 (2008).

12. Barrat, A., Barthélemy, M. \& Vespignani, A. Dynamical Processes on Complex Networks (Cambridge Univ. Press, 2008).

13. Cohen, R. \& Havlin, S. Complex Networks: Structure, Robustness and Function (Cambridge Univ. Press, 2010).

14. Bollobás, B. \& Riordan, O. The diameter of a scale-free random graph. Combinatorica 24, 5-34 (2004).

15. Cohen, R. \& Havlin, S. Scale-free networks are ultrasmall. Phys. Rev. Lett 90, 058701 (2003).
16. Erdös, P. \& Rényi, A. On the evolution of random graphs. Publ. Math. Inst. Hung. Acad. Sci 5, 17-61 (1960).

17. Bollobás, B. Random Graphs (Academic, 1985).

18. Watts, D. J. \& Strogatz, S. H. Collective dynamics of 'small-world' networks. Nature 393, 440-442 (1998).

19. Barabási, A-L. \& Albert, R. Emergence of scaling in random networks. Science 286, 509-512 (1999).

20. Lambiotte, R. et al. Geographical dispersal of mobile communication networks. Physica A 387, 5317-5325 (2008).

21. Bianconi, G., Pin, P. \& Marsili, M. Assessing the relevance of node features for network structure. Proc. Natl Acad. Sci. USA 106, 11433-11438 (2009).

22. Kosmidis, K., Havlin, S. \& Bunde, A. Structural properties of spatially embedded networks. Europhys. Lett. 82, 48005 (2008).

23. Song, C., Havlin, S. \& Makse, H. Self-similarity of complex networks. Nature 433, 392-395 (2005).

24. Gallos, L. K. \& Argyrakis, P. Absence of kinetic effects in reaction-diffusion processes in scale-free networks. Phys. Rev. Lett. 92, 138301 (2004).

25. Brockmann, D., Hufnagel, L. \& Geisel, T. The scaling laws of human travel. Nature 439, 462-465 (2006).

26. Bunde, A. \& Havlin, S. Fractals and Disordered Systems (Springer, 1991).

27. Official Airline Guide, http://www.oag.com/.

28. Shavitt, Y. \& Shir, E. Dimes: Let the Internet measure itself. SIGCOMM Comput. Commun. Rev. 35, 71-74 (2005).

29. Barrat, A., Barthélemy, M., Pastor-Satorras, R. \& Vespignani, A. The architecture of complex weighted networks. Proc. Natl Acad. Sci. USA 101, 3747-3752 (2004).

30. Boguñá, M., Papadopoulos, F. \& Krioukov, D. Sustaining the Internet with hyperbolic mapping. Nature Commun. 1, 62 (2010).

\section{Acknowledgements}

We thank ONR, DTRA, Deutsche Forschungsgemeinschaft (DFG), the Israel Science Foundation, and the European Project EPIWORK for financial support.

\section{Author contributions}

All authors contributed equally to the work presented in this paper.

\section{Additional information}

The authors declare no competing financial interests. Supplementary information accompanies this paper on www.nature.com/naturephysics. Reprints and permissions information is available online at http://npg.nature.com/reprintsandpermissions. Correspondence and requests for materials should be addressed to L.D. 